\title{
Code generator generation based on template-driven target term rewriting.
}

\author{
Annie Despland** Monique Mazaud* Raymond Rakotozafy** \\ *IN.RIA. Domaine de Voluceau, BP 105,78153 Le Chesnay CEDEX, France. \\ * *Université d'Orléans BP 6759, 45067 Orléans CEDEX, France and I.N.RIA.
}

\begin{abstract}
A major problem in deriving a compiler from a formal definition is the production of efficient object code. In this context, we propose a solution to the problem of code generator generation.

Our approach is based on a target machine description where the basic concepts used (access modes, access classes and instructions) are bottom-up hierarchically described by tree-patterns. These tree-patterns are written in an abstract tree language which is also used to write the intermediate program representation (input to the code generator).

The first phase of code production is based on access mode template-driven rewritings in which the program intermediate representation is progressively transformed into its "canonical form". The result is that each program instruction is reduced to a sequence of elementary instructions, each of these elementary instructions representing an instance of an instruction pattern.
\end{abstract}

The local and global optimizations phases as well as the storage management phase may be realized by multipass rewritings and attribute evaluations of the canonical form.

In the last phase of code production, each pattern instruction instance of the updated intermediate form is replaced by the corresponding instance of the associated pattern code .

\section{Introduction}

A compiler, in order to produce code, needs full knowledge of not only the syntax and the semantics of the source language but also of the structure of the target machine and the semantics of its instruction set. Therefore, each combination of source and target languages needs its own compiler. The profusion of new languages and computers leads to a constant demand for new compilers. Considerable research effort has been put into making the compiler construction as modular and automatic as possible. At present, automatic production of parsers from a BNF-like specification of grammars is widely known and used.

As the properties of the source and the target languages are quite different, it is necessary to introduce an intermediate representation (I.R).

Numerous works have developped techniques and tools for the implementation of retargetable code generators. Such tools should combine portability features and ease of writing for the compiler writer and make it possible :

- to clearly separate the description of a general technique from its application to a particular machine.

- to describe and achieve various code generation subtasks without imposing any particular ordering on them.

- to accept as input a description easy to deduce from the handbook.

The use of formal description of a target machine within a compiler writing system has given rise to several techniques. Some use a table-driven description of the instruction set, others use templates for each I,R primitive for which code has to be produced.

In the past few years, the code selection problem has received a lot of attention and has been relatively successfully dealt with in works by Cattell [Cat 77], Graham-Glanville [GG 78][GH 84], Ganapathi-Fischer [GF 82], 


\section{Ganzinger-Giegerich [Gan Gie 82]}

In contrast, the storage allocation phase of code generation seems to have been neglected with the exception of the PQCC system [LCHNRSW 79] [Lew 81], which gives a partial solution to this problem.

In all these works, the $\mathbf{I} . \mathbf{R}$ and the target machine specification are of a quite different nature.

- the Graham-Glanville approach is similar to the methods used for table-driven syntax analysis, and a target machine description is used to derive a parser. The overall structure of the code production algorithm is similar to the one of an LR(1) parser, which works on the linearized string of an intermediate tree and produces a sequence of instructions. It is necessary to design an I.R for each combination of source and target languages.

- Cattell uses TCOL (a universal tree language) for the I.R. From a complete specification of the instruction sef processor, tree templates are built which depict instructions. The code is emitted during a recursive top-down traversal of the I.R tree.

- Giegerich [Gie 85] describes the target machine as a signature of an algebraic abstract data type. The code generation is achieved by the production of a term of this abstract data type.

The main drawback of these systems is that the input I.R can not be parameterized. The solution proposed here overcomes this since it produces, as in the Perluette system [GDM 84] [Des 82], a compiler from a specification in three parts: a source language definition, a target language definition and the description of the implementation choices(see fig 1). As the source and target language definitions are algebraic data types, the I.R may be parameterized.

The target machine description is bottom-up and hierarchically structured in three levels:

- storage description (sets of available locations i.e registers, memory locations, stacks.)

- access modes description (various ways to access locations)

- instruction set description.

Generally speaking, the addressing mode semantics depends on the position of the corresponding operand. That is the reason why we have included the position in the definition of the concept of an access mode template. As an operand of an instruction may accept several access modes, the access modes are assembled in access classes. So the access classes are instruction parameters. The semantics of access modes and instructions is given in target data type terms. Once the correct I.R term is produced, the code generation will proceed in four steps (optimization tasks excluded):

- access modes templates pattern-matching. The I.R tree corresponding to an access mode template is rewritten in a sequence of elementary trees, each tree corresponding to an instruction template. An elementary tree corresponds to an intruction template instance.

When a term is complex, a subterm is loaded in a temporary variable; this requires the insertion of elementary universal store trees of temporary results in temporary storages.

We use universal store more efficiently than Cattell [Cat 77], because in our approach, temporaries are explicitly managed and have their own operators. This allows us to achieve the correct assignment of storage locations and an easy choice of the right mnemonic (for example, the choice between MOVB, MOVW and MOVL on the VAX is thus possible).

- instructions templates pattern-matching. The result of the first step is an $\mathbf{I} . \mathbf{R}$, whose leaves are instanciated access modes. In order to recognize an instruction template instance, it is necessary to verify that the access modes of this I.R belong to the corresponding access class of the instruction template.

At this point of the rewriting process, the $\mathbf{I} . \mathbf{R}$ is in its "canonical form". Tree transformations and attribute evaluations may be inserted to deal with optimization tasks.

- storage allocation. Each temporary occurrence is bound with the list of the allowed access modes and its type( register,memory word, memory byte ...). Considering the available storage locations and a 
preference order on the allowed access modes, this step allocates a physical storage to each temporary with the corresponding access mode.

- code production. Each instruction template is decorated with the corresponding assembly instruction mnemonic. Then code emission can be simply achieved by a left-right sequential tree traversal.

All the above steps concern the I,R tree. In particular, the allocation of storage locations transforms the tree leaves but has no immediate effect on code production: unlike Cattell, the possibility of choosing the right mnemonic is postponed until the end. Moreover, in our system, and contrary to the PQCC, the temporaries generated by the algorithm are set clearly apart and have their own operators. This enables them to be explicitly addressed. Optimizations specified by tree rewritings can take place at any time. Code production constitutes the ultimate phase of the global rewriting process of the I.R tree.

This solution allows a modular multi-pass code generation process with the different optimization tasks inserted as rewritings according to the compiler writer's wishes. Such an approach confers modularity and independence to our system as in the MUG2 system [GRW 77] [Gan Gie 82], while conserving the descriptive features of the Cattell and Graham-Glanville approaches.

Our solution to automatic production of code generators is complete in the sense that it tackles correctly both the code selection and the storage allocation problems.

This paper is divided into two sections. The first one presents the two specifications required as input to the code generator generator: a formal specification of the target machine and the I.R description.

The second one deals with the code generator generator itself: first, definitions of tree templates associated with the target machine components are given, then the I.R rewriting algorithm (which precedes the instructions selection phase) is developped. Finally, the looping and blocking problems are discussed.

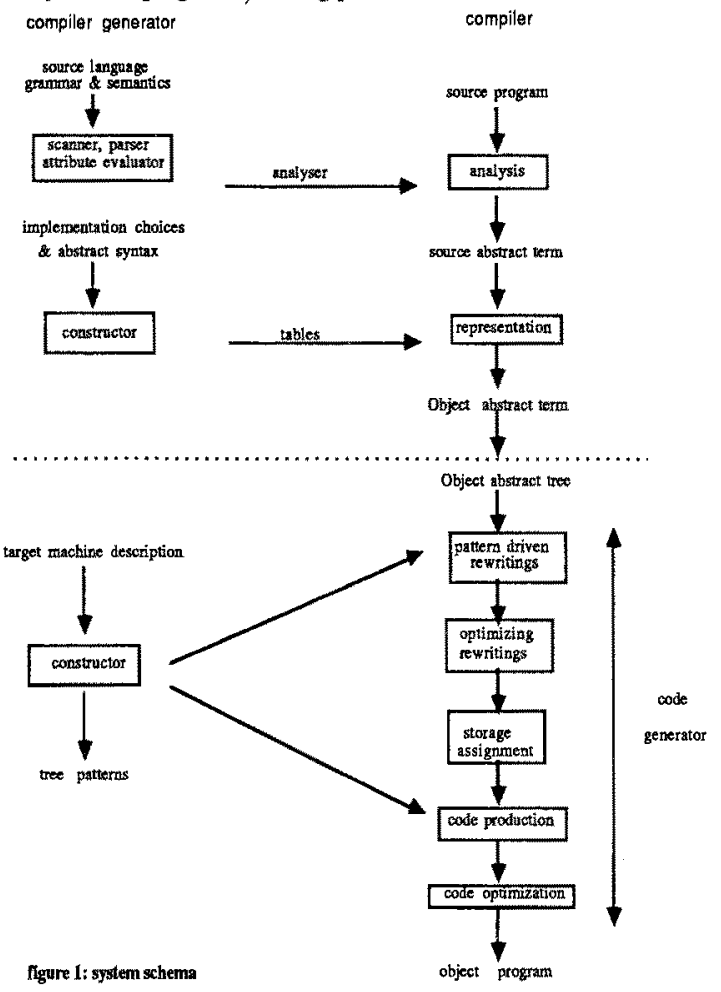




\section{Formal specification of the target machine}

Our goal is to propose a machine description formalism which is homogenous, exhaustive and correct. Our experience concerning the description of general-purpose registers machine such as PDP-11 [MRS 86] and VAX is encouraging. Concepts developped in this paper are illustrated by PDP-11 examples. The access modes and instructions formats are described in assembly language mnemonics. Then, the code generation phase produces an assembly language program.

\subsection{Components of the description}

The basic concepts used in the description are storages, access modes, operands and operations on the operands.

\subsubsection{Storage structuring}

The operand represented by a component of the physical storages set depends on the type of the operation applied to this operand. The reference to a register may represent either an access to the whole register, or an access to the least significant byte, or an access to the corresponding register pair.

So, we define two fundamental concepts: storage base and storage class.

A storage base is defined as the set of the smallest addressable units and is described by the following characteristics:

- the number of addressable units contained in it

- the size in bits of each unit

- the sequence which constitutes it

For the PDP-11, the smallest addressable memory location is the byte, so we define:

\begin{tabular}{|c|c|c|}
\hline \multicolumn{2}{|c|}{ Storage-base MEM } & . - Primary memory \\
\hline Cand & $=2 * 16$ & - cardinality of the set \\
\hline Size & $=8$ & - size in term of bits \\
\hline Set & $=\operatorname{MEM}[0 . .2 * * 16-1: 1]$ & \\
\hline
\end{tabular}

We define, as many storage classes as there are acceptable sequences of storage base elements.

A storage class is characterized by the following properties:

- the storage base

- the size of each element in bits

- the number of its elements

- the sequence of elements which constitute it

The primary memory of the PDP.11 may be considered as a sequence of bytes or as a sequence of two byte words. More precisely, a byte address allows to access the corresponding byte, but an even byte address allows to access the two byte word beginning at this address. Let us define the following two storage classes:

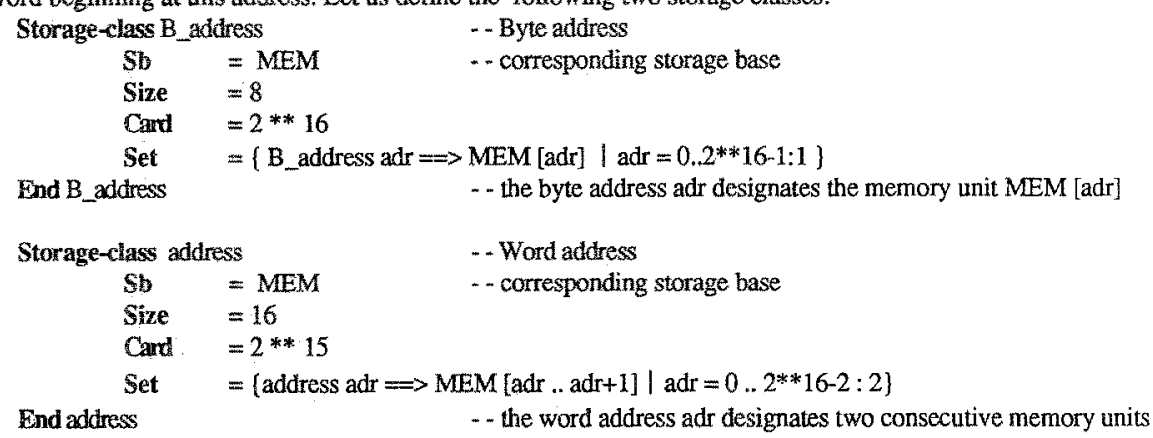

Similarly, the eight registers of the PDP-11 can be so addressed as to have access either to their least significant byte or to a whole register or even to a pair of consecutive registers. This leads to the definition of a register storage base as the set of bytes that make up the totality of registers and as many storage classes as there are acceptable sequences of register base elements. Let us define the storage class register: 


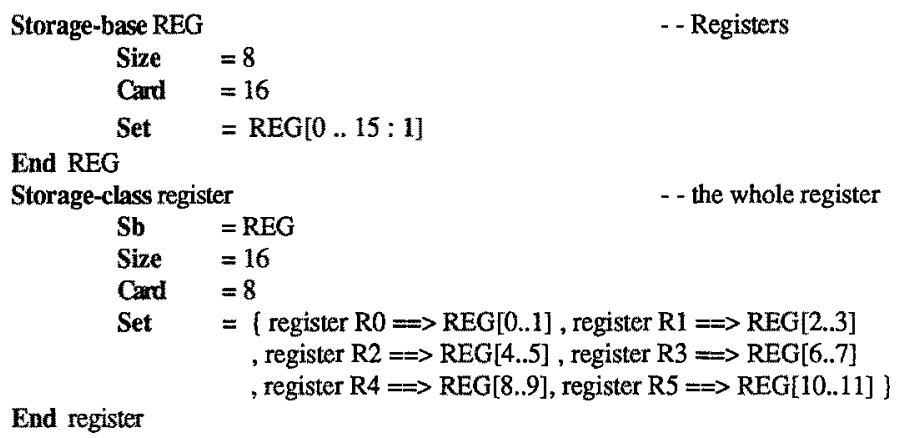

\subsubsection{Value Classes}

On any machine, there are instructions whose operands are immediate constants. Their value is generally stored in the address space of the instruction. We define value classes corresponding to allowed intervals for the operands to get homogeneous description. We suppose that there exists a value base INTEGER including all possible value classes. In the same way, we suppose that there exists a label value base named LABEL.

On the PDP-11, immediate value operand may be either a signed integer on a byte, or a signed integer on a word.

Value-class value

$$
\begin{array}{ll}
\text { Vb } & =\text { INTEGER } \\
\text { Size } & =8 \\
\text { Cand } & =65536 \\
\text { Set } & =\{\text { value val } \mid \text { val }=-32768 . .32767: 1\}
\end{array}
$$

End value

\subsubsection{Access modes}

A particular machine has several addressing modes. An addressing mode defines the access path to an operand. An operand is represented by an element of a storage class. Thus, for a given addressing mode of the machine, the specification includes as many access modes as there are associated storage classes. An access mode may be viewed differently depending on its particular use.

An access mode may be characterized by the following properties:

- the storage classes or value classes

- the format, i.e the syntax in the assembly language

- the template that describes the access path to the corresponding operand when it is on source position

- the semplate that describes the access path to the operand when it is on destination position

- the space or access time cost

- the side effect on the program counter

For the register addressing mode of the PDP-11, we define three access modes corresponding to the three storage classes : register, $B_{-}$register and $P_{-}$register. They allow access respectively to the content of the whole register, to the least significant byte of the register, and to the corresponding pair of registers.

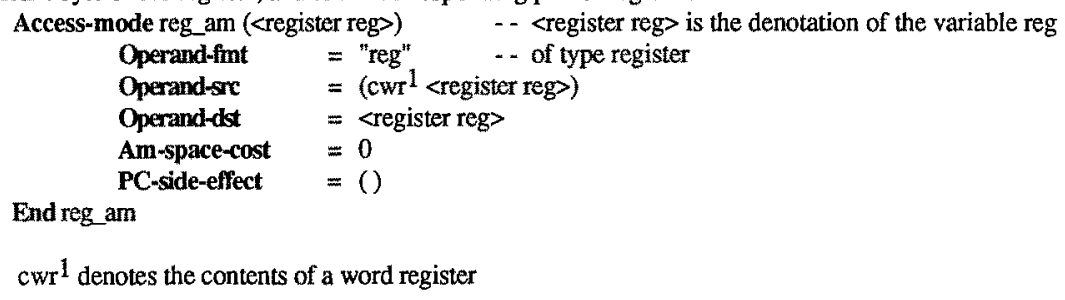

Note that throughout the paper, constants preceded by the name of their type are enclosed between " $<$ ", ">",

Let us consider the addressing mode autoincrementation of the PDP-11. It may be described as the access mode indirection followed by the side-effect: address incrementation of the register content. The autoincrementation relative to a word address may be described as follows: 


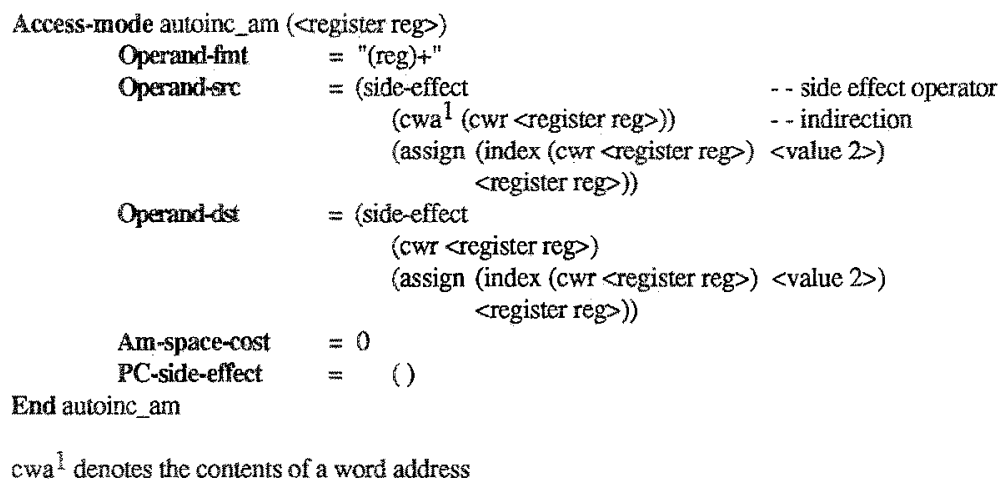

\subsubsection{Access classes}

In order to describe a machine instruction once only, we have assembled the access modes in access classes. Each class contains the access modes allowed for an operand instruction. These access classes allow a more concise specification of instruction models. The classes are not necessarily pairwise disjoint.

The multiply instruction MUL of the PDP-11 permits only a pair of registers as destination operand. So, it is necessary to describe an access class inchuding only the access class "pair of registers":

$$
\text { P_reg_access }=\text { (P_reg_am) }
$$

In the same way, the decrementation instruction with conditional branch SOB of the PDP-11 accepts only a register as source operand, so we define the access class: Reg_access = [reg_am]

The branch instruction accepts a label only as destination operand: Code_label_access = [code_label am

Except for multiply, the arithmetic and logical instructions of the PDP-11, accept an address or a register as destination operand. All access modes except immediate or register modes allow access to an address. The operand class Dynamic_access stands for all access paths to an address as destination operand. The access class Cell access defined below stands for all access paths to an operand in destination position.

$$
\text { Cell_access }=\text { (Dynamic_access, Reg_access) }
$$

The access class All access stands for all access paths to an operand in source position.

The concept of access class in our approach is more coherent than the operand class in Cattell. The concept of position (source or destination) included in the definition of operand classes leads the compiler writer to define two different classes for identical access modes sets. Moreover, as operand classes are parameters of instructions, it is possible to find in the source position an operand class related to the destination concept.

For instance, in Cattell's description of the PDP-11, we come across the instruction model:

$$
(<-\$ 1: D S T(+\$ 2: \text { DST } \$ 2: \text { SRC) })
$$

where S1: DST is used respectively in destination, then in source operand, whereas the operand class DST is exclusively related to the concept of destination.

This inconsistency does not happen with our concept of access class, because it does not include the concept of position. This concept appears in instruction modeis by means of specific operators.

\subsubsection{Instructions}

As for an access mode, an instruction may be viewed differently depending on its particular use. It may be characterized by the following properties: 
- the access classes

- the format, (the syntax in the assembly language)

- the template describing what is performed by the instruction in terms of operations of the abstract tree language

- the side effects on condition codes (if needed) and on the program counter

- the execution time cost

Since we always have an assembler, we choose to produce assembly code for the target machine. Hence, it is the assembler that deals with side effects on condition codes and program counter.

In our specification, the item "Side-effect" can be considered as a comment. It allows a more complete specification of the machine and could be used in binary code production.

As the reader can observe, to one instruction of the target machine corresponds one and only one instruction pattern in our description. A denotation of the access class (the name of the class coupled with a variable) is bound to one operand instruction template. The variable takes its values in the set of access modes template instances belonging to this class.

Let us consider the storage location clearing instruction CLR of the PDP-11:

Instruction clr $(<$ Cell_access AM $>$ ) $\cdots<$ Cell_access AM $>$ is the denotation of the variable AM of type Cell_access

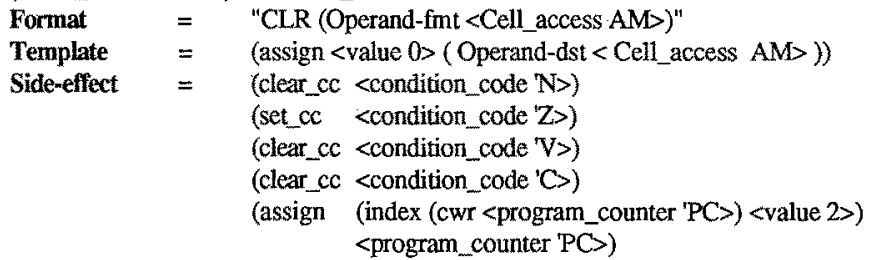

End clr

\subsection{Intermediate representation}

Let us summarize the specification mechanism of the code generator for a given target machine. The machine is specified by the description of available locations, access modes and instructions. As the locations are defined, names of sorts are associated to them. The access modes and instructions are specified by operators on locations. Thus, a set of operations with the corresponding dornains and co-domains can be defined.

Therefore the specification of the code generator includes two parts:

- the target machine description

- the object abstract data type related to the target machine.

In the framework of a translator writing system which works in three steps (see fig 1), the intermediate form input of the code generator is the object abstract term. It is the representation of the source abstract term which is the semantic value associated to the source program. The source term corresponding to the semantic value of a statement is a modification as defined in [GDM 84]. The representation of a modification of the source abstract data type is a modification in the object abstract data type.

The translation of the semantic value of the source progam into the semantic value of the object program is specified and proved [GDM 84] to be the representation of one abstract data type into another.

The basic I.R for the code generation process is the tree corresponding to the abstract target term. During attribute evaluation passes, this tree is decorated and transformed.

\section{Code generator writing system}

In order to emit the code related to an instruction, it is necessary to recognize an instance of an instruction template. As in the syntactic analysis process, an automaton is derived automatically. It is used by a target machine independant kernel which consists of an analyser and modules of rewriting and tree construction. 


\subsection{Templates and canonical forms}

The code generation process can carried out by matching the $\mathbf{I} . \mathbf{R}$ with instruction templates. This can be achieved by successive rewritings using the match of the I.R tree with an instruction template. In order to identify an instruction template, it is necessary to identify its children as access class templates. This can be done by means of access mode templates in a given position.

Conversely, the pattern matching process works in a bottom-up way. For each I.R instruction, it tries to identify access mode templates. Since the name of the access mode is needed to identify an access class, it is necessary to keep it in the $I . R$ tree. Thus an access mode tree is replaced by its canonical form: i.e, the tree whose root is the name of the access mode (parameterized by its position) and whose children are the parameters.

So the pattern matching process uses two kinds of templates: the access mode templates and the instruction templates.

\subsubsection{Access modes templates}

The system deduces the access mode ternplates from the specification, binding a term in a given position with the canonical form. Two kinds of templates will be stated, one for the access mode in the source position, and another for the access mode in the destination position. In order to emit the corresponding code, it is necessary to store the format; this is done by the means of the \&format attribute. By convention, attribute names are preceded by "\&".

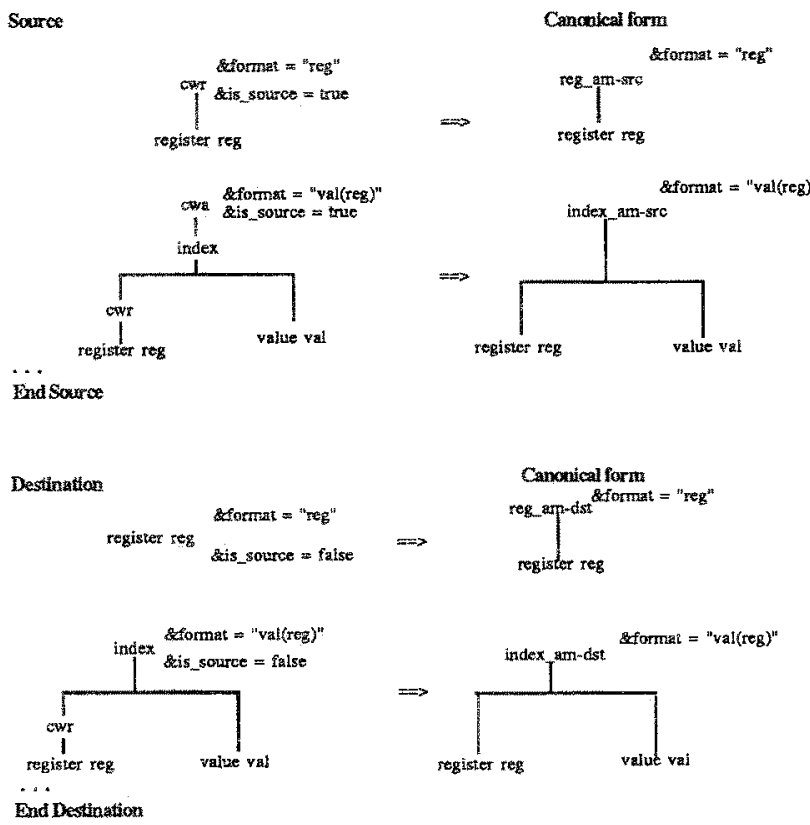

\subsubsection{Instruction templates}

An instruction template is a tree (decorated with the code attribute), the parameters of which are either constant denotations or access classes. The specification of a decrement, a move instruction and the templates associated to them are given below: 


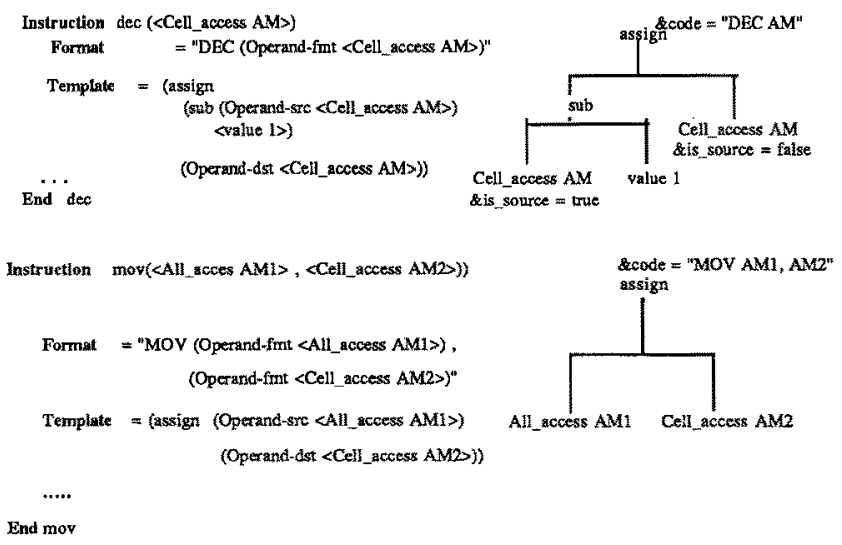

\subsection{Code generation}

The code generation process involves matching the I.R with instruction templates because an instruction template corresponds exactly to one assembly instruction. The code generator works in two steps:

- First, the top-down matching process of the I.R is carried out until the I.R is identified to an instruction template. The temporary storage allocation is done as needed.

- Second , the code is emitted after the temporaries have been assigned to actual storage locations.

\subsubsection{Intermediate representation rewriting}

\subsection{1.a Hypothesis}

1 - Since to recognize an instruction template, we have to recognize the access classes of its children, first all access modes must be identified. The largest possible subtree corresponding to an access mode will allow to produce better code ( "maximal munch" method). We are interested in the father of this tree. If it is not the root of a template, it will be necessary to store this address in a temporary location. This operation needs :

- the use of a universal temporary storage location associated with its access mode known by the code generator writing system; we shall denote them "temporary"and "temporary_am" respectively.

- the use of a store operator known by the code generator writing system and which can apply to each kind of temporary. The universal store " $\rightarrow$ " is used for this purpose, its rightmost operand is the destination.

In order to ensure compatibility with the I.R, the compiler writer must take into account all the above points. On the other hand, the system needs to know how to make the correspondence between " $\rightarrow$ " and the specific machine stores, when the corresponding instruction is emitted.

Trees are generated for temporary stores and they must be rooted in the sequence. An operator sequence must be available for the code generator writing system, by convention, we denote it by ";".

Thus, the compiler writer must specify equivalence declarations between the machine operators and the universal operators. This is done in an interface between the machine specification and the code generator writing system.

The temporary access mode is bound to the whole access mode selected for the management of temporary locations specified in a preference order. For the PDP-11, the equivalence declarations will be specified:

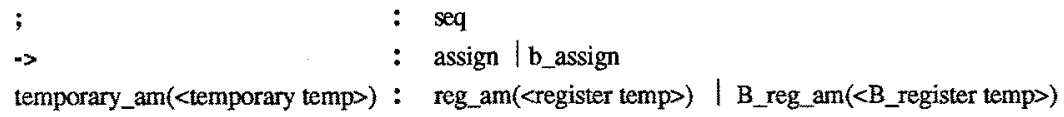


Here, the universal sequence operatne "i" is bound to the "seq" operator of the abstract data type. The universal store operator " $\rightarrow$ is bound to different assign operators of the abstract data type (assignment on words, assignment on bytes). The access mode on temporaries is bound to access modes allowing direct access to a register or to the memory.

2 - For each "generic" operation directed towards a storage base, the user must define as many operators as there are storage classes associated to the storage base. This constraint is used when dealing with the overlapping of access mode and instruction templates. For the access mode templates in source position, there is an access to either the contents of a storage class or to a value class. Thus, no access mode templates overlap in scurce position since there are as many access operators as there are classes.

In destination position however, overlapping may occur because there does not always exist an access operator to the contents of a storage class. In our rewriting algorithm, an access mode in destination position can only have as immediate ancestor a node of the abstract type "modification". Therefore, the preceding constraint (on the "generic" operation giving an operator for each storage class), is sufficent to handle the overlapping of templates.

The instruction template overlapping occur, in the case of machine instructions that have partially overlapping semantics. In this case, a criterion to solve the ambiguity may be the cost of the instruction .

\subsection{1.b Algorithm}

The algorithm belongs to the target machine independent kemel of the code generator. Access mode and instruction templates are considered as elements of a data base.

At present, the rewriting algorithm is applied to each element of a basic block.

For readability purposes, we assume that:

- there is no overlapping between instruction templates.

- there is no overlapping between instruction templates restricted to their root and their left child.

- we restrict ourselves to instructions the templates of which are rooted by binary operations of type modification which have no modification as domain.

We denote by the I.R tree for which we want to produce code. Steps of the algorithm are:

1. Constant expressions are corrputed.

2. The tree $\mathbf{T}$ is decorated with the attribute \&is_source = true everywhere but on the immediate right child.

3. Let us denote by $\mathbf{A}$, the largest possible subtree matched by an access mode $\mathbf{M}$. 


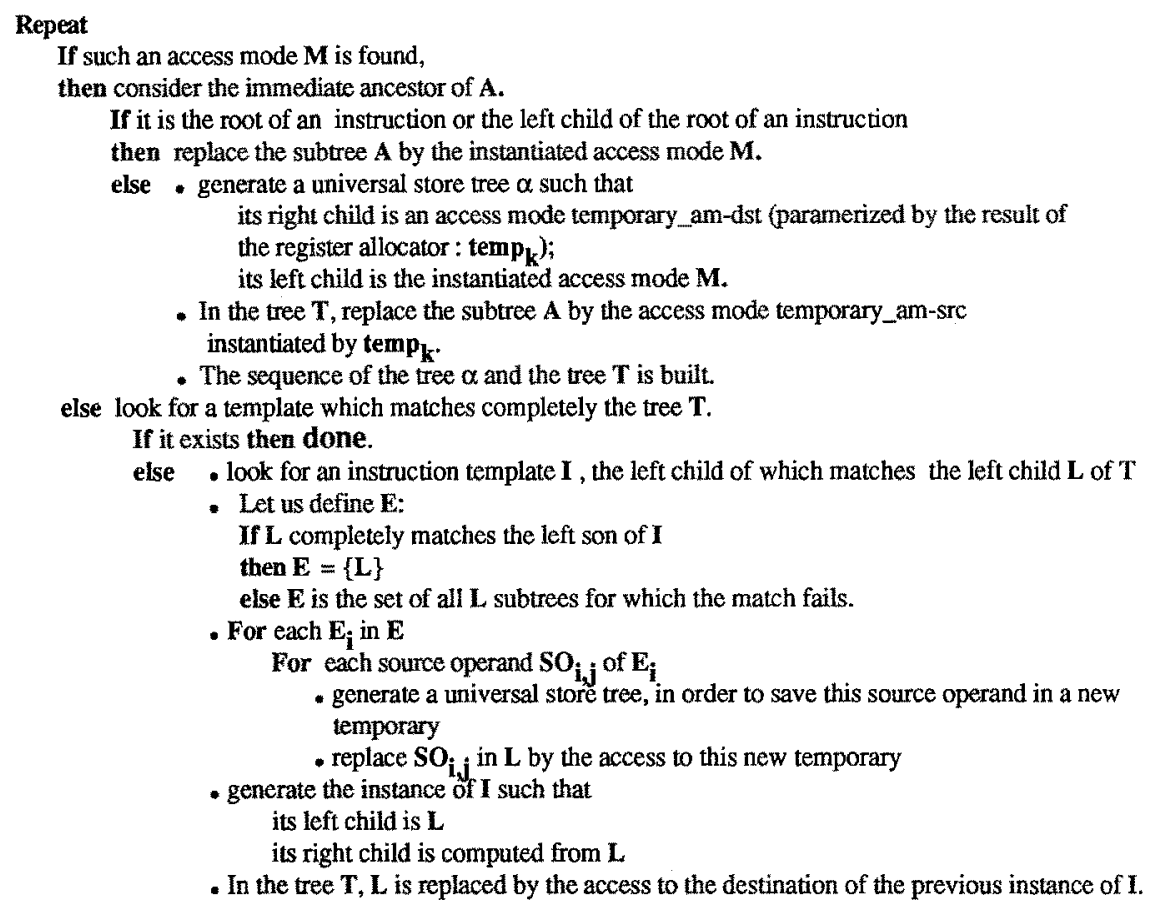

until done

\subsection{1.c Example}

Let us consider, in a procedural language, the I.R subtree corresponding to the push action of the actual parameter I - 1 , in a procedure call. A tree which designates a variable in a call can be written by indexing the offset of the variable to the base content of the procedure to which this variable is local. We assume that:

a) the register $R 5$ points to the beginning of the activation record of the current call.

b) -2 is the offset of I from the base of the procedure to which it is local (its father).

c) 2 is the offset of the father of I from the base of the current call

The following figures show successive rewritings. The access modes are designated by squares in dotted lines.

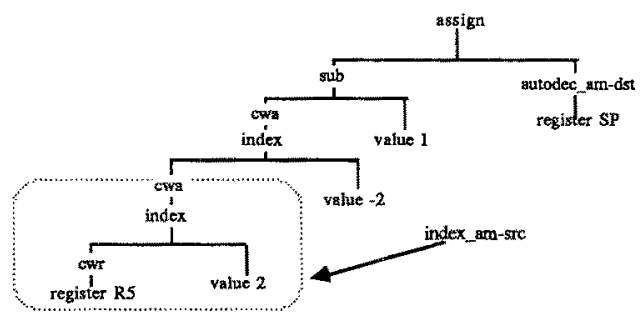

Step a: The access mode index am in source position is recognized, and the following sequence is built:

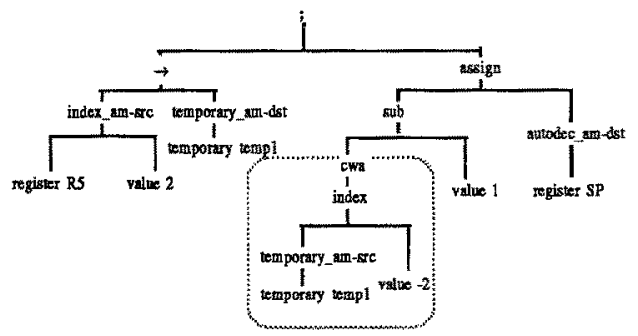


Step b: In order to recognize the index_am access mode, (in position sro), for the free in dotted lines, it is necessary that (temporary am-src <iemporary iempl>) be identified with (cwr <register reg>); because (temporary_am-src<temporary templ>) is associated to (reg_am-src <register templ>) via the interface and (reg_am-src <register templ>) is the canonical form of (cwr <register temp $1>$ ).

The recognition is made provided that the system records that the only allowed synonym of (temporary_am-src <temporary templ $>$ ) is (reg_am <register templ>).

The immediate ancestor is sub which is the root of the left child of an instruction template. So the system replaces the tree in dotted lines by (index am-src <temporary templ $>,<$ value $-2>$ )

Step $c$ : There is no more access mode instance in the sequence. No instruction template completely matches $Z$, the last tree of the sequence. So we look for an instruction template, the left child of which matches $Z$. The template which matches is:

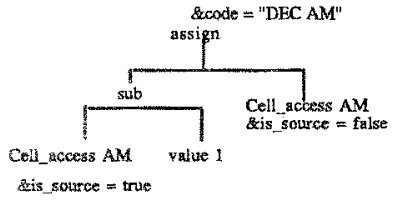

To obtain a successful unificakion, it is necessary to unify (index_am-src <temporary templ>, <value -2>) with $<$ Cell_access $A M>$. This is possible because the access mode index_am belongs to the access class Cell_access .

So the tree $\mathrm{Z}$ matches the decrementation template. A universal store tree is generated to save

(index am-src <temporary templ>, <value -2$\rangle$ ) in a new temporary. The instance of the decrementation template is generated. In the tree $Z$, the left child is replaced by the new temporary.

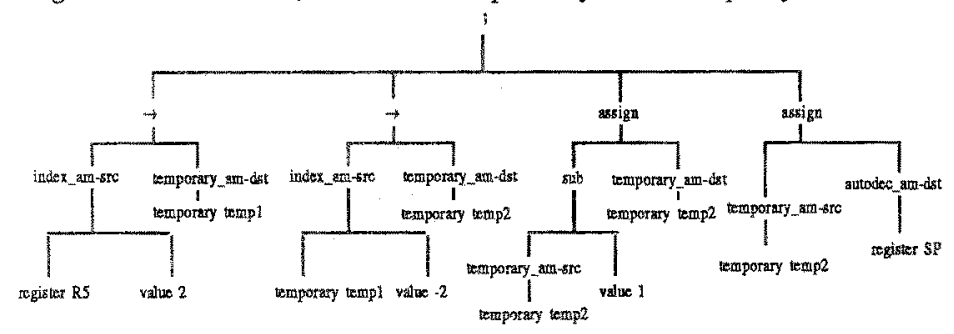

At this step of the rewriting, different optimization subtasks may be done by means of attributed tree transformations: common expression elimination, dead-live analysis, code motion based on loop invariant computations...

\subsubsection{Pattern-matching instruction selection}

Finally each subtree of the sequence is examined, and using the equivalence between the universal operators and the machine operators, the system recognizes instruction templates. The equivalence between the "assign" operator and the universal operator " $->$ " has been specified in the interface.

In the previous example, all subtrees but the third are assign templates and the third one is the decrement. Let us explain the pattern-matching process between the decrementation template and its instance in the preceding sequence.
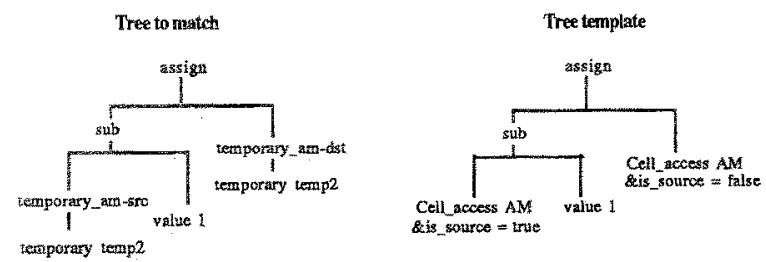
The pattern-matching succeeds only if:

1) the left chidren of such operators match only if:

a) AM takes the value (temporary_am-src < <emporary temp2>)

b) an access mode in the source position corresponds to the value "true" of the attribute \&is_source. This is true for the access mode temporary_am.

c) the operand class "Cell_access" includes "reg_am" and "relative_am", the only synonyms of (temporary_an <temporary temp2>) available at this step.

2) the right chidren of such operators match only if:

a) AM matches with the same access mode and its parameters.

b) an access mode in the destination position corresponds to the value "false" of the attribute \&is_source. This is true for the access mode temporary_am.

c) the same as 1). c)

Taking into account the available storage locations and the preference order specified in the interface, the storage assignment process chooses a synonym. If registers are available, the synonym chosen for (temporary_an <temporary temp2>) is (reg_am <register $R_{j}>$ ) where $R_{i}$ is the first free register.

If $\mathrm{R} 2$ is the first free register, the code emitted for the matched tree is : DEC R2.

If no register is available, the synonym will be ( relative_am <data_label $L_{i}>$ ) where $L_{i}$ is the label of a memory area. If 'TMP1 is the label result, the code emitted will be : DEC TMP1.

In the example of 2.2.1.c, let us suppose that only the register R1 is avatlable. As the only synonym for (temporary_am <temporary temp1>) is (reg_am <register templ>), templ will take the value R1. Then the synonym of (temporary_am <temporary temp2>) will be (relative_am <data_label temp2>). The result of the rewriting is:

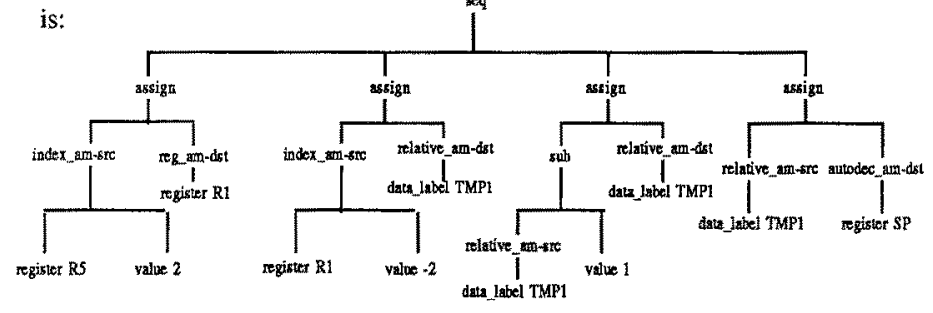

$\begin{array}{lll}\text { Thus, the code emitted is: } & \text { MOV } & \text { 2(R5) , } \\ \text { MOV } & -2(\mathbf{R 1}) & \text {, TMP1 } \\ \text { DEC } & \text { TMP1 } & \\ \text { MOV } & \text { TMP1 } & - \text { (SP) }\end{array}$

The compiler writer can insert optimization modules which transform attribute trees before emitting code.

\subsubsection{Looping and blocking}

In our system, looping cannot occur, because at each loop of the algorithm the depth of the tree decreases.

In our algorithm, if an instruction is recognized, it succeeds, otherwise there is a blocking. This situation arises if no address template or no instruction template can be recognized.

Blocking occurs when the environment of an operator of the object abstract data type is not compatible with the one required for this operator in the access mode or instruction templates. This situation comes from a source language semantic description which cannot be translated directly in the object language.

Let us consider the tree $P$ of the I.R describing the action of pushing the address of a reference parameter. The offset of the parameter from the base of the current call is -2 . 


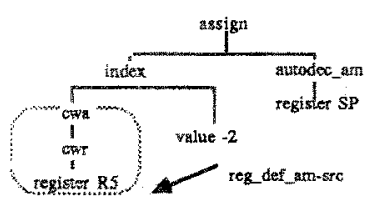

Step 1: The access mode "reg_def_am" is recognized in the source position. The following sequence is obtained:

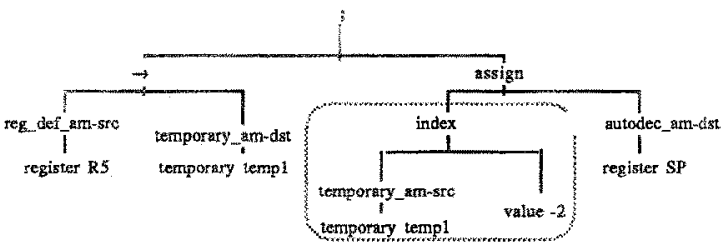

Step 2: The subree enclosed in doned lines does not correspond to an access mode in the source position. No template matches completely the tree P. No template, the root of which is "assign" matches the left child of P: it is a blocking situation. It corresponds to the fact that no indexation can occur in the source position of an assign instruction. A transformation specified in the interface may be :
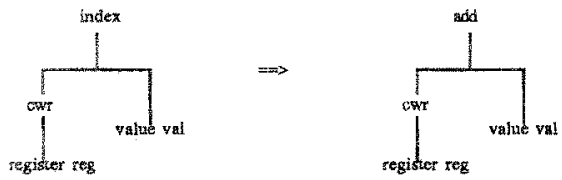

The following tree is obtained by applying these transformations and by using the equivalences between

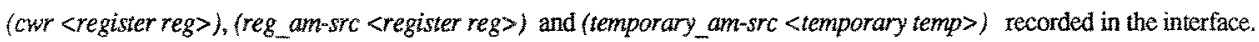

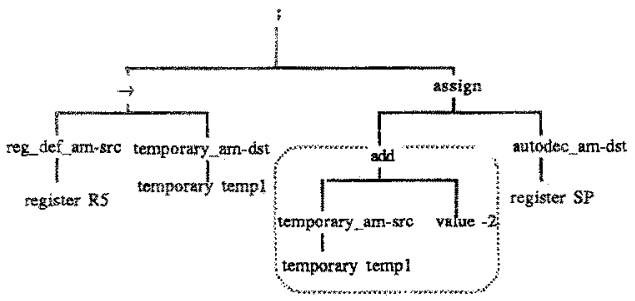

The only synonym allowed for (temporary_am <temporary templ $>$ ) is (reg_am<register templ $>$ ). As "add" and "sub" have similar descriptions, the algorithm runs as for "sub" from the step cof the example of 2.2.1.c , and the following tree is obtained:

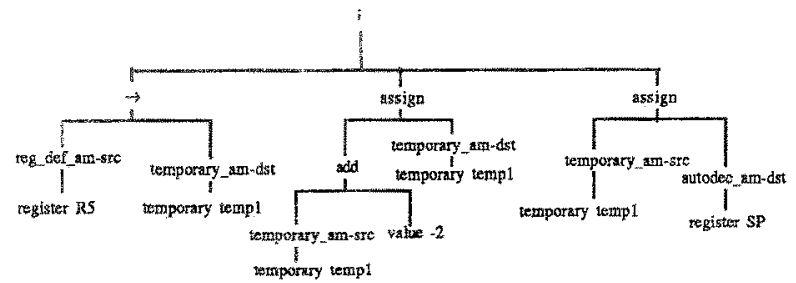


After register assignment , temp1 becomes $\mathrm{R} 2$, and the code emitted is :

$\begin{array}{lll}\text { MOV } & (\mathbf{R} 5), & \mathbf{R 2} \\ \text { ADD } & \# 2 & \\ \text { MOV } & \mathbf{R 2} & \text { R2 } \\ \text { (SP) }\end{array}$

\section{Conclusion}

In our approach, the code generator generation process is summarized by the following essential points:

- the description of the machine should be straightforward, i.e easy to deduce from the handbook. Now, the ease with which we achieved complete specifications of different kinds of machines: Motorola-6809, PDP-11, VAX-11, RIDGE has been very convincing. Each specification needed only a few days for completion.

- there is a clear-cut distinction between the description of a general technique, and its application to a particular machine. The code generation algorithm is based on tree rewriting, the tree templates are deduced from the machine specification.

- the modularity of the code generation subtasks is achieved. In the code generation algorithm, the clear-cut partition between the storage location assignment and the code selection processes is made possible, owing to the use of general temporary locations coupled with their own operators.

Our solution allows a multi-pass code generation process, which is genuinely modular. The different optimization tasks are considered as rewritings, that the compiler writer insert at wish. Thus we propose dealing with optimizations as rewritings of attributed trees.

Bibliography

[Cat 77] Cattell R. G. G. "A Survey and Critique of some Models of Code Generation" Carnegie-Mellon University, Computer Sciences Department, Technical Report, 1977.

[Cat 80] Cattell R. G. G. "Automatic Deriyation of Code Generators from Machine Description" ACM Transactions on Programming Languages and Systems, Vol. 2, No.2 p173..199, April 1980.

[Des 82] Deschamp Ph. "PERLUETTE : a compiler producing system using abstract data types" Proceedings of International Symposium on Programming, Turin, April 1982.

[Don 82] Donzeau-Gouge V.. "Les raisons des choix dans la définition formelle du langage ADA" Thèse d'état Paris VII 1982.

[GDM 84] Gaudel M. C., Deschamp Ph, Mazaud M. "Compiler Construction From High Level Specification". Automatic Program Construction Techniques. Macmillan Inc, 1984.

[Gan Gie 82] Ganzinger H, Giegerich R. "A truly Generative "Semantics-Directed Compiler Generator" Proceedings of the SIGPLAN 82 Symposium on Compiler Construction, ACM SIGPLAN Nº. 17, 6, June 1982.

[GF 82] Ganapathi M., Fischer C.N. "Descriptive-Driven Code Generation Using attributed Grammars" Conference of the Nineth Annual ACM Symposium on Programming Languages.

[GG 78] Graham S.L, Glanville R. S. "A New Method for Compiler Code Generation" Conference Record of the Fith Annual ACM Symposium on Principles of Programming Languages, p231 240, January 1978.

[GH 84] Graham S. L., Henry R. R. et AL "Experiment with a Graham-Glanville Style Code generator" Proceedings of the SIGPLAN 84 Symposium on Compiler Construction, ACM Not 19, 6, June 1984.

[Gie 85] Giegerich R. "Logic specification of Code Generation Techriques" Lecture Notes in Computer Science. Programs as data objects, Coperihague 17-19 October 1985.

[GRW 77] Ganzinger H., Ripken K., Wilhelm R. "Automatic Generation of Optimizing Multipass Compilers" IFIP77, North-Holland Publishing Company p535.540, 1977. 
[Kro 75] Kron H. H. "Tree Templates and subtree transformational grammars" PhD-thesis Univ of Cal , Santa Cruz, 1975.

[Ho O'D 82] Hoffmann C.M, ODonnell M.J. "Pattern Matching in Trees" JACM vol 29 No 1, Jan 1982, pp 68-95.

[CHNRsW 79] Leverett B.W., Cattell G.G., Hobbs S.O., Newcomer J.M., Reiner A.H., Schatz B.R., Wulf W.A. "An Overview of the Production Quality Compiler-Compiler Project" CMU-CS-79-105.

[Lev 81] Leverett B.W. "Register Allocation in Optimizing Compilers" CMU-CS-81-103.

[Lun 83] Lunell H. "Code Generator writing systems" Software Systerns Research Center \$-581 83 Linköping, Sweden.

[Mad 83] Madelaine E. "Système d'aide à la preuve de compilateurs" Thèse de 3ème cycle, Université de Paris VII, Seprembre 1983.

[MRS 86] Mazaud M., Rakotozafy R., Szumachowski-Despland A. "Code generator generation based on template-driven target term rewriting" rapport de recherche INRIA RR-582, 1986. 